Research Paper

\title{
Galectin-1 Promotes Vasculogenic Mimicry in Gastric Cancer by Upregulating EMT Signaling
}

\author{
Xiaolan You ${ }^{\bowtie}$, Qinghong Liu, Jian Wu, Yuanjie Wang, Jiawen Dai, Dehu Chen, Yan Zhou, Yanjun Lian \\ Department of Gastrointestinal Surgery, Hospital Affiliated 5 to Nantong University (Taizhou People's Hospital), Taizhou, Jiangsu province, China \\ $\triangle$ Corresponding author: Xiaolan You, Department of Gastrointestinal Surgery, Hospital Affiliated 5 to Nantong University (Taizhou People's Hospital), \\ Taizhou, Jiangsu province, 225300,China e-mail:006586@yzu.edu.cn \\ (C) The author(s). This is an open access article distributed under the terms of the Creative Commons Attribution License (https://creativecommons.org/licenses/by/4.0/). \\ See http://ivyspring.com/terms for full terms and conditions.
}

Received: 2019.02.01; Accepted: 2019.08.31; Published: 2019.10.17

\begin{abstract}
Background: Galectin-1 (Gal-1) expression was positively associated with vasculogenic mimicry (VM) in primary gastric cancer (GC) tissue, and that both Gal-1 expression and VM in GC tissue are indicators of poor prognosis. However, whether Gal-1 promotes VM, and by what mechanismsremains unknown.

Methods: To investigate the underlying mechanisms,wound healing assay, proliferation assay, invasion assay, and three-dimensional culture were used to evaluate the invasion, metastasis and promoted VM formation effects of the Gal-1. We monitored the expression level of sociated proteins in GC tissues, cell lines in vitro and nude mice tumorigenicity in vivo by immunohistochemistry and western blot.

Results: Gal-1 overexpression significantly promoted the proliferation, invasion, migration, and VM formation of MGC-803 cells. Gal-1 was associated with E-cadherin and vimentin in vitro and in clinical samples. The epithelial-to-mesenchymal transition (EMT) induced in MGC-803 cells by TGF- $\beta 1$ was accompanied by Gal-1 activation and promotion of VM formation, while knockdown of Gal-1 reduced the response to TGF- $\beta 1$, suggesting that Gal-1 promotes VM formation by activating EMT signaling. Overexpression of Gal-1 accelerated subcutaneous xenograft growth and facilitated pulmonary metastasis in athymic mice, enhanced the expression of EMT markers, and promoted VM formation in vivo.
\end{abstract}

Conclusion: Our results indicated that Gal-1 promotes VM in GC by upregulating EMT signaling; thus, Gal-1 and this pathway are potential novel targets to treat VM in GC.

Key words: Galectin-1; Vasculogenic mimicry; Gastric cancer; Epithelial-to-mesenchymal transition

\section{Introduction}

Gastric cancer (GC) is one of the leading causes of death worldwide, with a high incidence and a high rate of lethal malignancies in China [1]. In the past two decades, although great progress has been made in the diagnosis and therapy of GC, the overall survival rate remains unsatisfactory, especially in advanced disease [1, 2]. Anti-angiogenesis treatment offers new hope for the treatment of GC, especially in chemotherapy-resistant patients [3]. It was long believed that angiogenesis via the sprouting of endothelial vessels from existing ones was the exclusive form of tumor vascularization, and anti-angiogenesis therapy was considered as a promising method to treat tumors $[3,4]$. However, with the wide application of angiogenesis inhibitors in clinical practice, it was established that the effect of antiangiogenic drugs was limited [5]. This indicated that other modalities supply tumor nutrition in addition to endothelial vessels.

In 1999, Maniotis et al. [6] first reported an endothelial-independent vascular channel, which formed with highly aggressive and metastatic melanoma cells, and this formation was termed vasculogenic mimicry (VM). Soon afterwards VM was 
identified in multivalent malignant tumors, especially in poorly differentiated malignancies, including gallbladder carcinoma [7], hepatocellular carcinoma [8], ovarian cancer [9], small cell lung cancer [10], glioblastoma [11], pancreatic duct carcinoma [12], and gastric adenocarcinoma [13]. VM comprises a basement membrane, lined externally by tumor cells and a lack of endothelial cells in the internal lining [6, 14]. Based on the endothelial cells visualized by CD34 immunohistochemical staining and the basement membrane visualized by Periodic acid-Schiff (PAS) histochemical staining, this PAS-CD34 double staining showed that the endothelial-dependent vasculature was CD34-PAS double positive, while VM was CD34 negative/PAS positive. Recent research indicated that VM might play an extremely important role in the biological behavior of human malignant tumors [15].

Galectin-1 (Gal-1) is a $\beta$-galactoside-binding protein that belongs to a 15 -member protein family called the galectins [16]. Among the 15 members of lectin family, Gal-1 appears to be the major player in cancer biology, which has stimulated significant research interest [16]. Recent studies have found that extracellular Gal-1 is overexpressed in the stroma between tumor cells in multivalent malignant tumors [16-20]. In addition, increased expression of Gal-1 correlated with a variety biological behaviors of malignant tumor, including cellular aggregation, cellular apoptosis, metastatic spread of cancer, tumor immunity, and angiogenesis [16-23]. However, little is known about the association between Gal-1 and VM in GC.

Previously, we reported that Gal-1 expression was positively associated with VM in primary GC tissue, and both Gal-1 expression and VM in primary GC tissue are indicators of poor prognosis after gastrectomy [24]. However, whether Gal-1 promotes $\mathrm{VM}$, and by what mechanisms, remains unknown. In this study, we explored the role of Gal-1 in the development of $\mathrm{VM}$ and its underlying mechanisms in GC.

\section{Materials and methods}

\section{Tumor tissue samples}

As previously reported, 127 patients with gastric adenocarcinoma were enrolled in our study, no preoperative neoadjuvant chemotherapy or radiotherapy had been administered. All patients underwent radical gastrectomy at the Department of Gastrointestinal Surgery, Taizhou People's Hospital, Jiangsu province. GC tissues for immunohistochemistry (IHC) and histochemical staining were formalin-fixed. This study was approved by the Clinical Research Ethics Committee of our institute (TZRY-EC-12-068).

\section{Cell culture and lentiviral transduction}

The human gastric adenocarcinoma cell line MGC-803 was obtained from the Type Culture Collection of the Chinese Academy of Sciences (Shanghai, China). The cells were maintained with Roswell Park Memorial Institute medium (RPMI; Thermo Scientific Hyclone, Waltham,MA , USA) supplemented with 10\% FBS (fetal bovine serum; Thermo Scientific HyClone,), $100 \mathrm{U} / \mathrm{ml}$ penicillin, and $100 \mathrm{mg} / \mathrm{ml}$ streptomycin (GIBCO, Grand Island, Waltham, MA, USA), under conditions of $37^{\circ} \mathrm{C}$ in a humidified atmosphere containing $5 \% \mathrm{CO}_{2}$.

Lentiviral vectors carrying green fluorescent protein (GFP) and a puromycin resistance gene for Gal-1 overexpression and knockdown were commercially constructed by Genechem Co. Ltd. (Shanghai, China). MGC-803 cells were seeded on 6-well plates at $5 \times 10^{4}$ cells per well before lentiviral transduction. Cells were transduced with the appropriate lentiviral vector containing at a multiplicity of infection (MOI) of 10 in the presence of $10 \mu \mathrm{g} / \mathrm{ml}$ polybrene (Sigma-Aldrich, Temecula, CA, USA). The appropriate fresh medium replaced the medium after incubation at $37^{\circ} \mathrm{C}$ for $12 \mathrm{~h}$. Puromycin (Sigma-Aldrich) was added to select for stably transduced cells at concentration of $2 \mu \mathrm{g} / \mathrm{ml}$ after incubation for $48 \mathrm{~h}$. Stable transductants were cultured with puromycin at $0.5 \mu \mathrm{g} / \mathrm{ml}$. Transduction efficiencies were evaluated $72 \mathrm{~h}$ after transduction by counting GFP positive cells under a fluorescent microscope (OLYMPUS-U-HGLGPS-IX73); positive transduction was further confirmed by quantitative real-time reverse transcription PCR (qRT-PCR) and western blotting.

\section{RNA extraction and real-time PCR}

Total RNA was extracted using an RNeasy Mini Kit (Invitrogen, Waltham, MA, USA). First strand cDNA synthesis was performed using a reverse transcription kit (Takara, Shiga, Japan) and the real-time PCR analyses were conducted on an iQ5 Multicolor Real-Time PCR Detection System (Bio-Rad, Hercules, CA, USA) using the SYBR Green dye (Roche Diagnostics, Mannheim, Germany). The GAPDH gene was used as an internal control, and the data are shown as the fold change. The experiment was performed in triplicate. Primers for Gal-1, Vimentin (VIM), E-cadherin (CDH1), and GAPDH are shown in Table 1. 
Table 1. Primers used for qRT-PCR

\begin{tabular}{lll} 
Gene & Forward sequence & Reverse sequence \\
\hline Gal-1 Ensembl:ENSG00000100097 & GCTGAACCTGGGCAAAGACAG & GTTGAGGCGGTTGGGGAACTT \\
Vimentin Ensembl:ENSG00000026025 & TGAATACCAAGACCTGCTCAA & ATCAACCAGAGGGAGTGA ATC \\
E-cadherin Ensembl:ENSG00000039068 & TCGTCACCACAAATCCAGTG & CATTCACATCAAGCACATCC \\
GAPDH Ensembl:ENSG00000111640 & TGACTTCAACAGCGACACCCA & CACCCTGTTGCTGTAGCCAAA
\end{tabular}

\section{Western blotting analysis}

Total cell extracts and nuclear extracts were prepared using an extraction kit (Beyotime, Shanghai, China). $12 \%$ sodium dodecyl sulfate-polyacrylamide gel electrophoresis (SDS-PAGE) gels were used to separate $20 \mu \mathrm{g}$ of cell lysates and the separated proteins were transferred to a nitrocellulose membrane (GE Healthcare Life Sciences, Pittsburgh, PA, USA). Blots were probed with rabbit monoclonal antibody against Gal-1 (Cell Signaling Technology, Danvers, MA, USA), rabbit monoclonal antibody against vimentin (Cell Signaling Technology), rabbit monoclonal antibody against anti-E-cadherin (Cell Signaling Technology), or mouse monoclonal antibody against GAPDH (Kang Cheng, Shanghai, China) antibodies at a dilution of 1:2000. Horseradish peroxidase (HRP)-conjugated goat anti-mouse immunoglobulin and HRP-conjugated goat anti-rabbit immunoglobulin were used as a secondary antibody at a dilution of 1:2000. The West Pico chemiluminescent Substrate (Pierce, Carlsbad, CA, USA) was used to visualize the immunoreactive protein bands, and densitometric image analysis software (Image Master VDS; Pharmacia Biotech) was used to quantify the visualized protein bands. The level of GAPDH was used as an internal reference. All experiments were performed in triplicate.

\section{Proliferation assay}

The Cell Counting Kit-8 (CCK-8) assay was used as aqualitative index of cell proliferation.We plated ten thousandcells in 96-well microplates; CCK-8 (Dojindo, Kumamoto, Japan) was used according to the manufacturer's protocol. Briefly, we added $10 \mu \mathrm{L}$ of CCK-8 solution to each well, then incubated the samples for $1 \mathrm{~h}$, and measured the absorbance at 450 $\mathrm{nm}$. All experiments were performedin triplicate.

\section{Cell invasion assay}

24-well Transwell units with polycarbonate filters (pore size, $8.0 \mu \mathrm{m}$; Corning, New York, USA) were used to measure the invasive ability of GC cells. In brief, the upper Transwell inserts were first coated with $100 \mu \mathrm{l}$ Matrigel basement membrane (BD Biosciences, San Diego, CA, USA), and then $1 \times 10^{5}$ cells in $100 \mu \mathrm{L}$ of serum-free RPMI medium were seeded on it. Medium (600 $\mu$ l) containing 10\% FBS was placed in the lower chamber as a chemoattractant. Cells were allowed to migrate at 37
${ }^{\circ} \mathrm{C}$ for $24 \mathrm{~h}$; non-invasive cells were removed with a cotton swab. $4 \%$ paraformaldehyde was used to fix the filters, and the cells were stained with a $0.05 \%$ crystal violet solution, and counted under a microscope with six randomly-selected fields at 100x magnification for each sample. Invasion assays were performed in triplicate.

\section{Wound-healing assay}

When cells reach 80-90\% confluence in a 6-well plate, a sterile plastic pipette tip was scored across the cell surface to create a wound. The plates were washed three times to remove cellular debris using phosphate-buffered saline (PBS), and then incubated at $37{ }^{\circ} \mathrm{C}$ with serum-free medium containing $10 \mu \mathrm{g} / \mathrm{mL}$ mitomycin $\mathrm{C}$ (to block proliferation). The wound was photographed at $0 \mathrm{~h}$ and $48 \mathrm{~h}$. All experiments were performed in triplicate.

\section{Three-dimensional culture}

A 24-well plate was coated with $200 \mu 1$ growth factor-reduced Matrigel (BD Biosciences, San Diego, CA, USA), which was allowed to polymerize at $37^{\circ} \mathrm{C}$ for $1 \mathrm{~h}$, after which $1 \times 10^{5}$ cells suspend in $600 \mu \mathrm{l}$ of medium containing 10\% FBS were plated on the surface of the gel and incubated at $37^{\circ} \mathrm{C}$ for $24 \mathrm{~h}$; three wells were provided for each group. Cells were then photographed under an inverted microscope (OLYMPUS-U-HGLGPS-IX73).

\section{Histological examination and IHC evaluation}

IHC was performed according to our previous report [24]. Slides were incubated with primary antibodies against Gal-1 (1:200), E-cadherin (1:200), or vimentin (1:200); the other steps and staining scores of Gal-1 were the same as in our previous report[24]. All specimens were stained in three sections using each antibody. E-cadherin and vimentin staining was defined as positive or negative. At a magnification of $400 \times$, ten fields of each section were randomly selected under the microscope. Two independent pathologists blinded to the patient's clinical status assessed the results. The evaluation of staining result was accordance with the previous report[25]. The staining cells percentage was scored as 0 for $0-5 \%$; 1 for $6-25 \%$; 2 for $26-50 \%$ and 3 for $50-100 \%$, the staining intensity was scores as 0 point for negative; 1 point for weak intensity; 2 points for moderate 
intensity and 3 points for strong intensity. The sum scores $\geq 3$ points were considered as positive.

\section{CD34-PAS dual staining and VM evaluation}

CD34-PAS dual staining and VM evaluation were performed according to our previous report [24]. CD34-PAS dual staining of subcutaneous GC tumors and lung metastases was performed on formalinfixed, paraffin-embedded tissue. Sections were cut at $4-\mu \mathrm{m}$ thickness, and all specimens were stained in three sections using each antibody. At a magnification of 200x, ten fields of each section were randomly selected under the microscope to distinguish the positive or negative VM.

\section{Animal models}

Subcutaneous GC implantation and lung metastasis models were established in five-week-old male athymic mice respectively. Mice were bought from the Comparative Medicine Centre of Yangzhou University (Yang Zhou, JiangSu, China), and the experiments were approved by the Ethic Committee of Yang Zhou University (YZU-EC-JS2352), breeded mice in the laminar flow cabinet under pathogen-free conditions. MGC-803 cells overexpressing Gal-1 (OEGal-1), with Gal-1 knock-down (KD- Gal-1), or wild-type control(WC) MGC-803 cells were separately inoculated into the right sides of the back or the tail vein of the athymic mice $\left(2 \times 10^{6}\right.$ cells/mouse; $n=$ 6 /group). The diameters of the subcutaneous tumors were measured every three days. The mice in subcutaneous group were sacrificed on day 21 and the mice in lung metastasis group were sacrificed on day 50. The subcutaneous GC tumors and lung metastases were harvested and subjected to histological examination using hematoxylin and eosin (H\&E) staining, IHC, or CD34-PAS dual staining.

\section{Statistical analysis}

SPSS 16.0 (SPSS, Chicago, IL, USA) was used to conduct the statistical analysis. Continuous variables were expressed as the mean $\pm \mathrm{SE}$ and Student's $t$-test was used to compare between groups. The Chi-squared test was applied to compare dichotomous variables. The nonparametric Spearman-Rho method was used to analyze the correlations. In all analyses, $P<0.05$ was considered statistically significant.

\section{Results}

Gal-1 promotes invasiveness and VM-formation potentiality of GC cells

To investigate whether Gal-1 contributes to VM, two lentiviruses were constructed to establish MGC-803 cell lines with stable overexpression of the
Gal-1 gene (OE-Gal-1) and Gal-1 silenced (KD-Gal-1), which were confirmed by detecting the GFP signal (Figure 1A and 1D), qRT -PCR (Figure 1B and 1E), and western blotting (Figure $1 \mathrm{C}$ and $1 \mathrm{~F}$ ). Compared with MGC-803 cells infected with the knockdown control virus (KD-CON), knockdown of Gal-1 resulted in MGC-803 adopting a fusiform shape (Figure 1A). OE-Gal-1 cells exhibited more elongated architecture than MGC-803 infected with the overexpression control virus (OE-CON) (Figure 1D). As shown in Figure 2A, OE-Gal-1 cells exhibited a significantly enhanced migration capacity compared with cells infected with the OE-CON lentiviruses and the wild-type control, while KD-Gal-1 cells lost their ability to migrate. By the method of Proliferation assay, we observed that the proliferation of MGC-803 cells was increased with elevated Gal-1 expression (Figure 2B). Gal-1 overexpression in MGC-803 increased cell invasiveness, while knockdown of Gal-1 in MGC-803 cells destroyed their ability to invade (Figure 2C). Interestingly, Gal-1 overexpression increased the cells' capacity for tube-formation in MGC-803 cells, while KD-Gal-1 cells lost their ability to form tube-like structures (Figure 2D). These results suggested that Gal-1 plays an important role in the promotion of invasion and the induction of VM formation in GC.

\section{Gal-1 expression was elevated in an EMT model}

It has been reported that the EMT contributes to tumor cell plasticity and is the key step during VM formation [26-28]. As such, we hypothesized that Gal-1 contributes to VM formation by inducing EMT. We treated MGC-803 cells with $10 \mathrm{ng} / \mathrm{ml}$ transforming growth factor beta 1 (TGF- $\beta 1$ ) for 24 hours, which caused the cells to exhibit a more elongated architecture than MGC-803 control cells (Figure $3 \mathrm{~A}$ ). In addition, the TGF- $\beta 1$-treated cells showed substantially reduced levels of the epithelial marker E-cadherin, and increased the levels of the mesenchymal marker vimentin both in mRNA and protein level (Figure 3B and 3C). Interestingly, treating MGC-803 cells with TGF- $\beta 1$ could significantly increase the expression of Gal-1 (Figure 3C), which suggested a possible role for Gal-1 in the EMT of GC cells. Meanwhile, typical tube-like structures increased in the 3-dimensional culture in the TGF- $\beta 1$ induced EMT model (Figure 3D). This finding suggested a possible role of Gal-1 in inducing EMT and promoting VM formation in GC.

\section{Gal-1 induced VM in GC cells by upregulating EMT signaling}

Based on the finding that Gal-1 is increased in MGC-803 cells in the EMT model treated with 

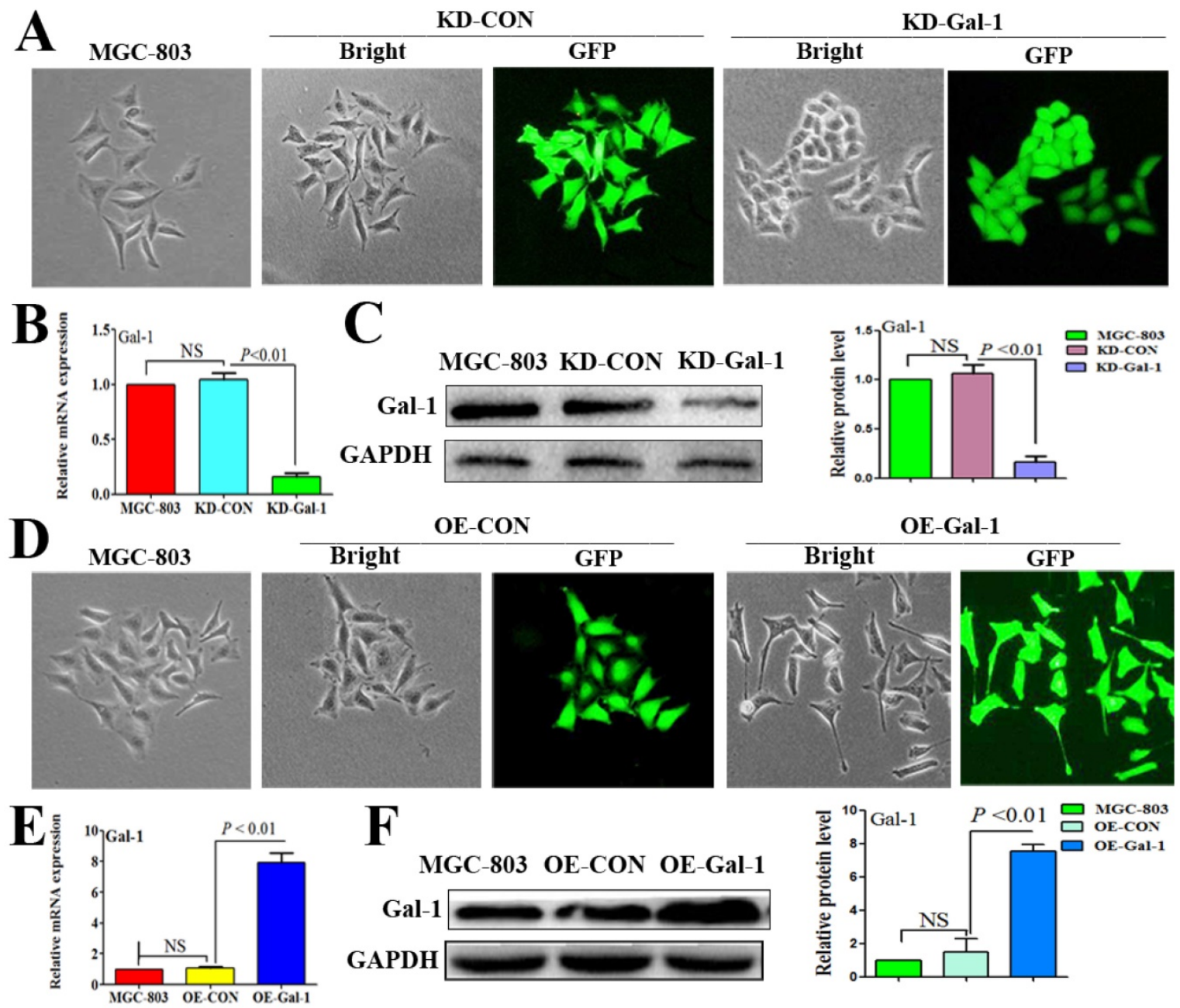

Figure 1. Established MGC-803 cell lines with stable silencing of Gal-1 (KD-Gal-1) and with overexpression of Gal-1 gene (OE-Gal-1) (A) Transfection of KD-Gal-1 lentiviruses induced morphological changes in MGC-803 cells, as confirmed by the GFP signal (magnification: $\times 200$ ). (B and C) Stable knockdown of Gal-1 expression in MGC-803 cells was assessed using QRT-PCR and western blotting. (D) MGC-803 cells overexpressing Gal-1 (OE-Gal-1) exhibited a more elongated architecture than MGC-803 infected with the overexpression control virus (OE-CON), (magnification: $\times 200)$. (E and F) Stable overexpression of Gal-1 in MGC-803 cell line was assessed using qRT-PCR and western blotting. The bars represent the mean of three independent experiments \pm SD.

TGF- $\beta 1$, and that the TGF- $\beta 1$-induced EMT model promoted typical tube-like structures in MGC-803 cells, we next determined whether overexpression of Gal-1 promoted the VM formation of GC cells by inducing EMT. MGC-803 cells with Gal-1 knockdown (KD-Gal-1) exhibited significantly increased E-cadherin and decreased vimentin levels (Figure 4A and 4B), while overexpression of Gal-1 (OE-Gal-1) in MGC-803 cells decreased the E-cadherin level and increased the vimentin level (Figure 4C and 4D). We then assessed the TGF- $\beta 1$ treatment in MGC-803 cells and its counterpart which with Gal-1 knockdown and treat with TGF- $\beta 1$ in MGC-803 cells, including the EMT marker proteins and VM formation ability. Similar to previous studies, treatment of MGC-803 cells with TGF- $\beta 1$ for 24 hours increase the level of vimentin and decreased that of E-cadherin (Figure 4E and $4 \mathrm{~F}$ ). However, Gal-1 knockdown cells lost their responsiveness to TGF- $\beta 1$ induction (Figure $4 \mathrm{E}$ and $4 F)$. Meanwhile, stimulation with TGF- $\beta 1$ increased the ability of MGC-803 to form VM, while the ability of VM formation was decreased in the Gal-1 knockdown cells (Figure 4G). These results suggested that Gal-1 induced VM-formation in GC cells by upregulating EMT signaling.

\section{Gal-1 associates with EMT-related biomarkers in $\mathbf{G C}$ tissues}

To further determine whether Gal-1 can promote EMT and induce VM in GC tissue, we examined EMT-related markers in GC tissue using IHC, and identified the VM using CD34/PAS double-staining. We identified positive vimentin staining in both the tumor stroma and GC cells (Vimentin +) in 42 cases, and E-cadherin was decreased in those samples (Figure 5A). In addition, vimentin was expressed only in mesenchymal tissues (Vimentin -) in the remaining 85 samples, and E-cadherin was expressed only in epithelial tissues (Figure 5B). Moreover, the IHC scores of Gal-1 in primary tumors in the 42 
vimentin-positive cases were higher than those in 85 vimentin-negative cases (Figure 5D). There was a significant association between the IHC score of Gal-1 and the expression of vimentin $(P<0.01)$. In the 127 cases, CD34-PAS double staining revealed VM in 29 cases $(22.8 \%)$; the remaining 98 cases were VM-negative (77.2\%) (Figure 5C). Furthermore, 17/29 VM-positive cases also showed vimentin overexpression, while only 25/98 VM-negative cases had vimentin overexpression (Figure 5E). There was a significant association between the expression of vimentin and VM $(P=0.01$,). These results suggested that EMT is associated with VM formation in GC, and that Gal-1 contributes to this process.

\section{A}

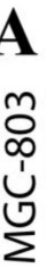

$\mathrm{Oh}$
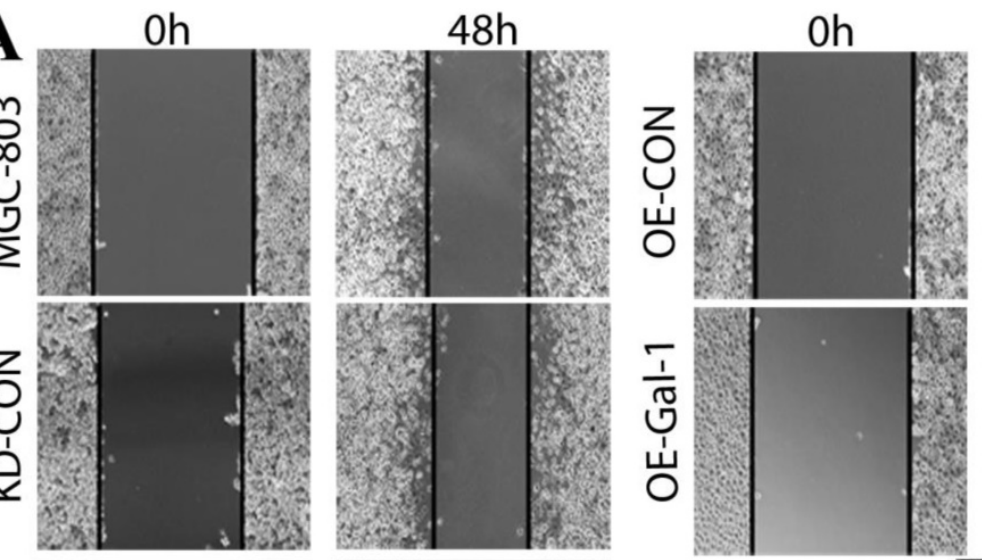

Oh

$48 \mathrm{~h}$
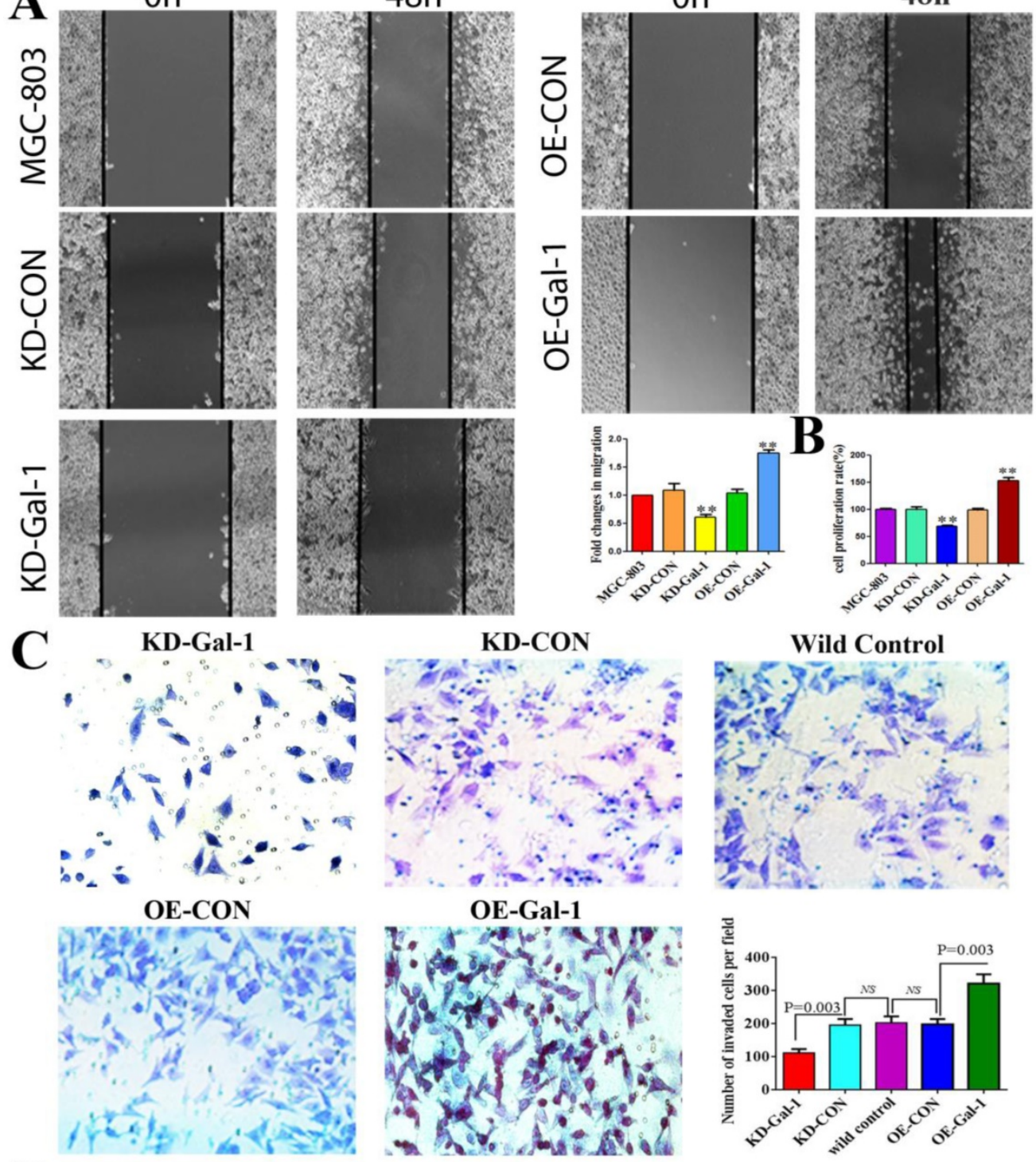

OE-Gal-1
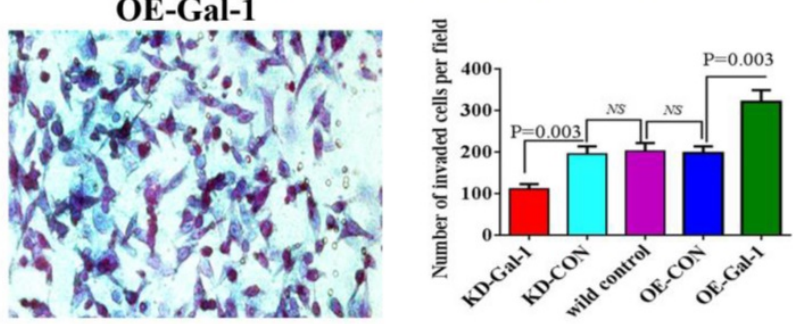

D
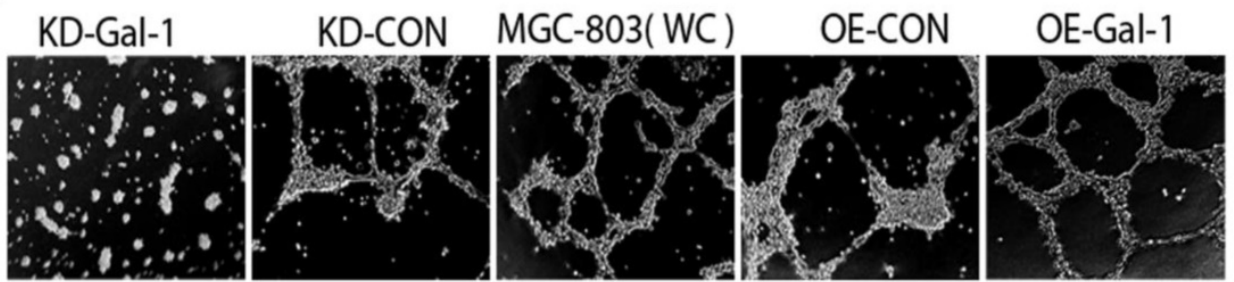

Figure 2. Gal-1 promotes proliferation, invasiveness and VM formation potential of gastric cancer cells. (A) Gal-l overexpression in MGC-803 cells exhibited significantly enhanced migration capacity after $48 \mathrm{~h}$ in a wound assay, while knockdown of Gal- $I$ in MGC- 803 cells destroyed their ability to migrate. Magnification: $\times 40$. $*$ P $<0.05$, $* * P<0.01$. These results are presented as mean $\pm S D$ of three independent experiments. (B) Gal-1 promotes the proliferation of MGC-803 cells. (C) Matrigel invasion assay showing MGC-803 cells with Gal-1 overexpression or knockdown; the numbers of invaded cells were quantified in six randomly selected fields at $\times 200$ magnification. Bars represent the mean number of invaded GC cells (six fields/sample). (D) Matrigel three-dimensional culture showing that Gal-l overexpression increased the capacity for tube-formation in MGC-803 cells, while knockdown of Gal-1 in MGC-803 cells destroyed their ability to form tube-like structures. Original magnification, 40x, $\mathrm{n}=3$. 


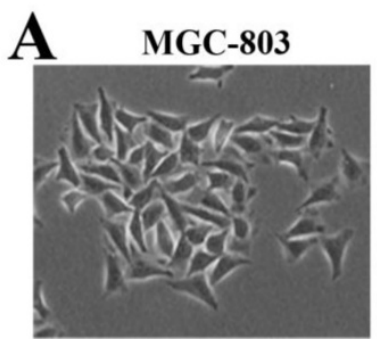

B

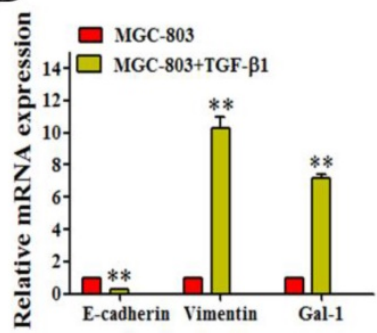

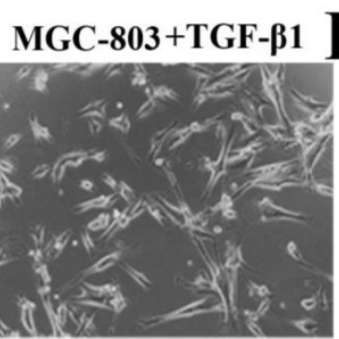

C

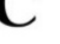

E-cadherin

Vimentin

Gal-1

GAPDH

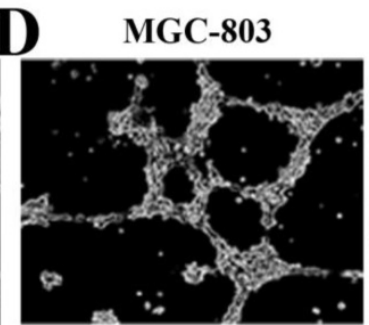

MGC-803+TGF-ß1

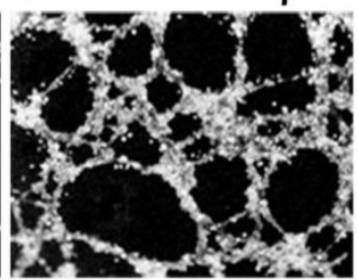

MGC-803

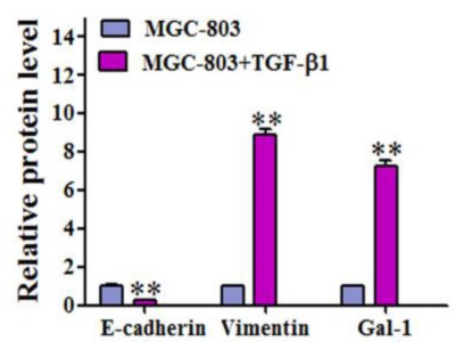

Figure 3. Gal-1 expression was elevated in a TGF- $\beta 1$-induced EMT model (A) MGC- 803 cells treated with $10 \mathrm{ng} / \mathrm{ml}$ TGF- $\beta 1$ for 24 hours exhibited a more elongated architecture; original magnification, 200x (B and C) qRT-PCR and Western blot showing an increased Vimentin level, decreased E-cadherin level, and increased Gal-1 level. * $P$ $<0.05$, $* * P<0.01$. The bars represent the mean of three independent experiments \pm SD. (D) Typical tube-like structures increased in 3 -dimensional culture in the TGF- $\beta 1$-induced EMT model; original magnification, 40×. $n=3$.

A

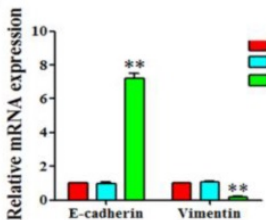

C

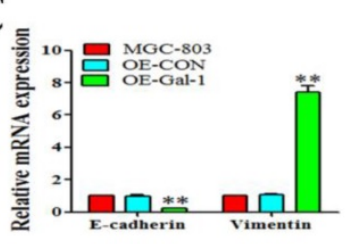

B
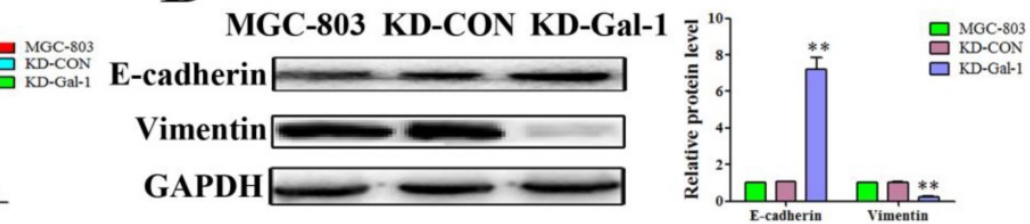

D

E-cadherin

Vimentin

GAPDH

IGC-803 OE-CON OE-Gal-1

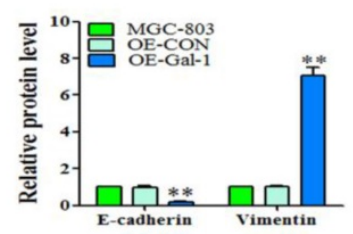

E

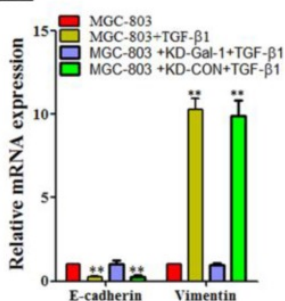

E-cadherin

Vimentin

GAPDH
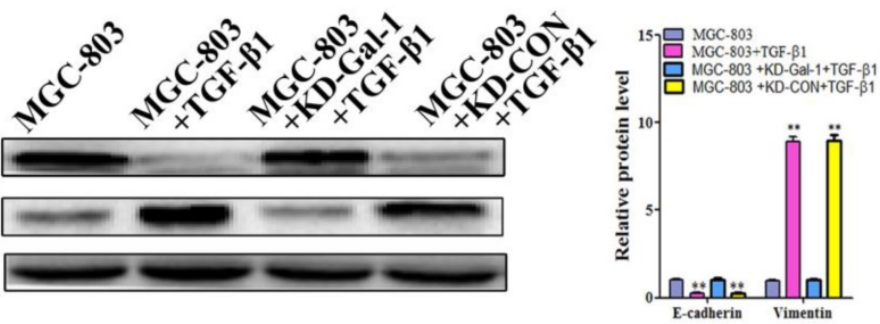

G

MGC-803

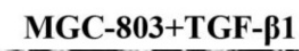

MGC-803+KD-Gal-1
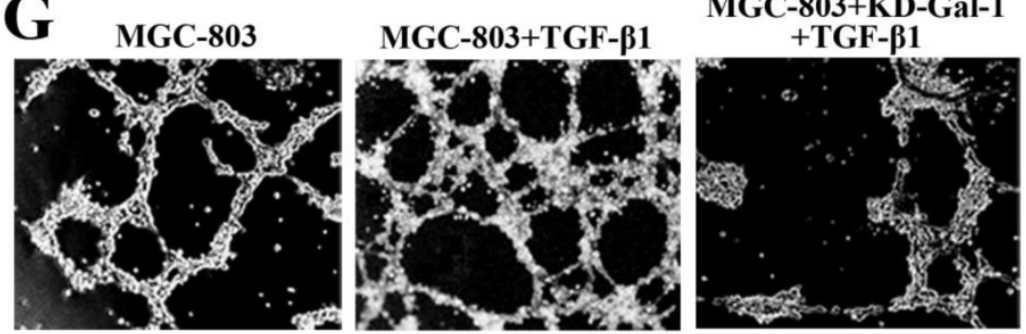

MGC-803 + KD-CON + TGF- $\beta 1$

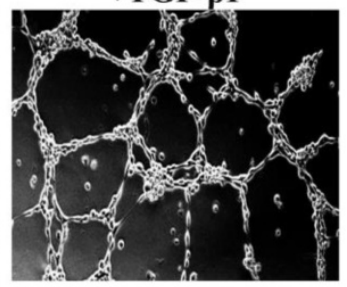

Figure 4. Gal-1 overexpression led to EMT and form VM in gastric cancer cells (A and B) MGC-803 cells with Gal-I knockdown (KD-Gal-1) exhibited significantly increased of E-cadherin and decreased vimentin. ( $C$ and D) Over expression of Gal-I (OE- Gal-1) in MGC-803 cells decreased E-cadherin expression and increased vimentin expression. (E and F) Gal-I knockdown caused MGC-803 cells to lose their responsiveness to TGF- $\beta 1$ induction. $* P<0.05$, ** $P<0.01$. The bars represent the mean of three independent experiments \pm SD. (G) Stimulation with TGF- $\beta 1$ increased the ability of MGC-803 to form VM, while Gal- $I$ knockdown MGC-803 cells failed to form VM. $n=3$. 


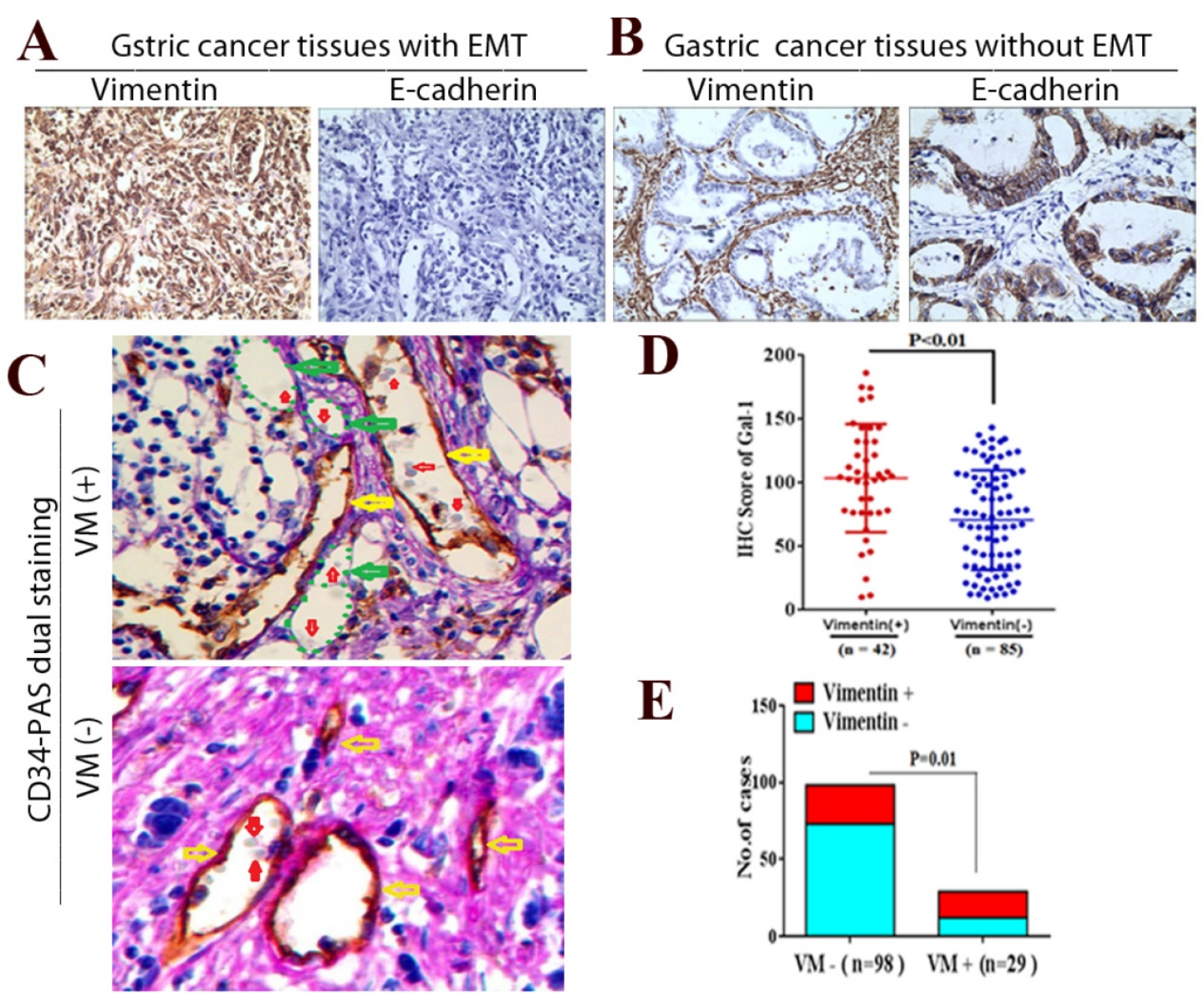

Figure 5. Gal-1 associates with EMT-related biomarkers in GC tissue. (A and B) Vimentin and E-cadherin are identified with IHC in gastric cancer tissues with EMT and without EMT. (C) CD34-PAS staining showing endogenous cell-dependent vessels (yellow arrows),VM (green dotted line) and red blood cells(red arrow) in GC specimen with VM and without VM. Original magnification: $\times 400$. (D) The Gal-1 IHC scores in primary tumors with EMT were significantly different to Gal-1 score in primary tumors without $\operatorname{EMT}(P<0.01)$. (E) EMT status was compared between vimentin positive GC cases and vimentin negative cases $(P=0.01)$.

\section{Gal-1 promotes VM in vivo}

Through in vitro experiments and clinical analysis, we demonstrated that Gal-1 promotes VM formation in GC through an EMT-mediated process. Subcutaneous GC implantation and lung metastasis models in athymic BALB/c mice $(n=6 /$ group) were generated to further determine the relationship between Gal-1 and GC growth, metastasis, VM formation, and the EMT-related markers. Twenty-one days after subcutaneous GC implantation, tumors in the Gal-1 overexpression group (OE) were larger and heavier than wild-type control group (WC), and the tumors in the Gal-1 knockdown groups (KD) were significantly smaller and lighter than those in the wild-type control group (Figure 6A, B; $P<0.01$ ). From day 6 onward, the tumor volume in the KD-Gal-1 group was significantly lower compared with that in the wild-type control, and the volumes of the tumors in the OE-Gal-1 group were significantly higher after day $15(P<0.01$ and $P<0.05$; Figure $6 \mathrm{C})$. Compared with the wild-type control group, Gal-1 expression was observed to be elevated in the OE-Gal-1 group using immunostaining, while KD-Gal-1 group was negative for Gal-1 expression (Figure 6D). CD34/PAS staining indicated that VM was significantly increased in the OE-Gal-1 group, and immunostaining showed positive vimentin staining in both the tumor stroma and tumor cells, while E-cadherin staining was negative in those samples. By contrast, VM was not observed in the tumor tissues in the KD-Gal-1 and wild control groups, and vimentin was detected only in mesenchymal tissues (Figure 6E).

Fifty days after the generation of the lung metastasis models, larger and more extensive pulmonary metastases were found in every one in the OE-Gal-1 group, 4 cases had pulmonary metastasesin the wild-type control group, while the KD- Gal-1 group did not have any pulmonary metastases $(P=$ 0.002; Figure 7A). H\&E staining showed pulmonary metastases in the OE-Gal-1 group and the wild-type control group (Figure 7A). CD34-PAS double staining and immunostaining were used to examine the VM and EMT-related markers in the pulmonary metastases. CD34/PAS staining indicated that VM was significantly increased in the pulmonary metastases tissues in the OE-Gal-1 group (Figure 7B). Immunostaining showed that vimentin was positive 
in both the tumor and tumor stroma in the OE-Gal-1 group, and E-cadherin was negative in the tumor tissues (Figure 7C). These results suggested that Gal-1 plays an important role in GC invasion, metastasis, and VM-formation, and that EMT potentially contributes to this process.
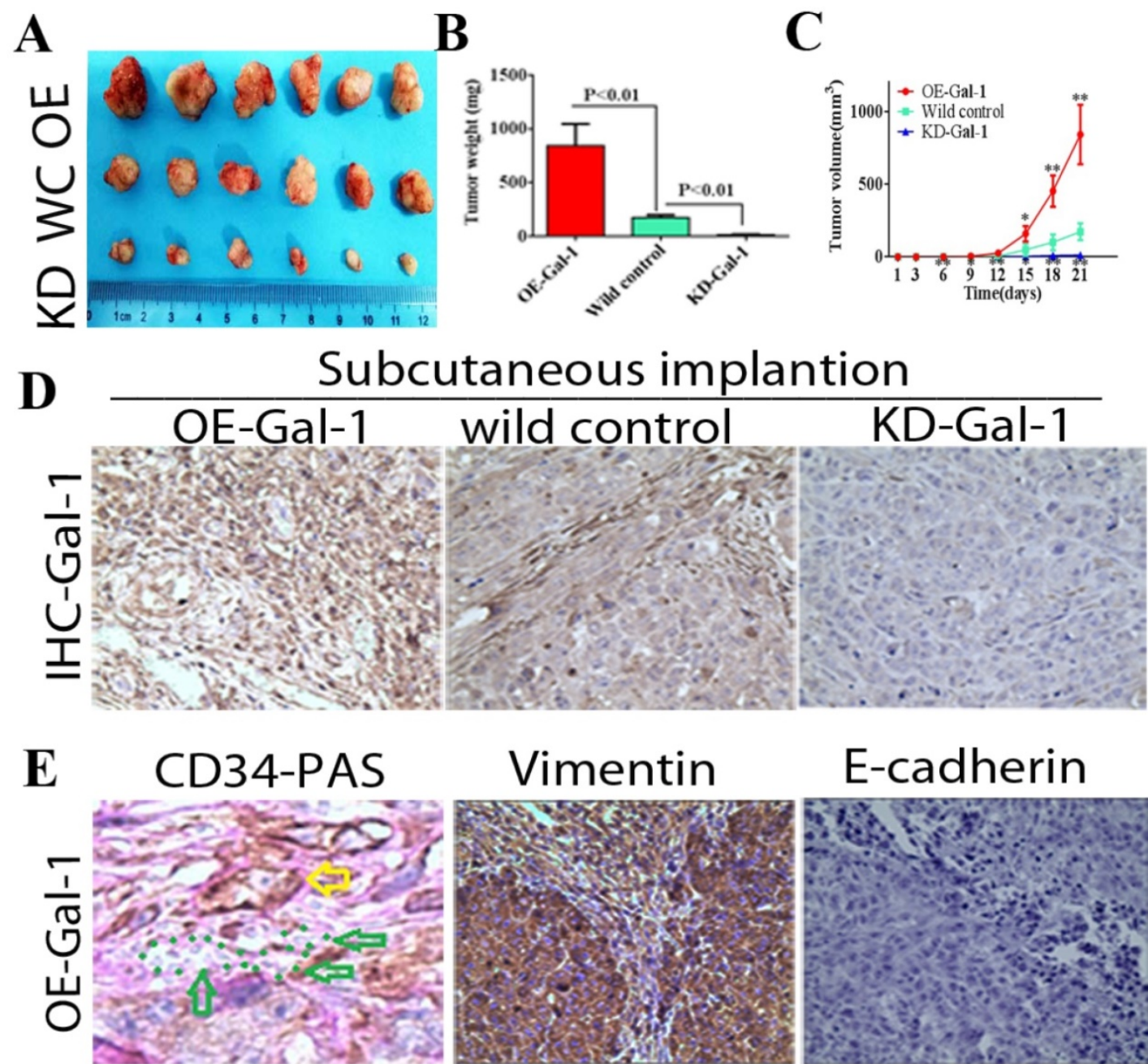

\section{E-cadherin}
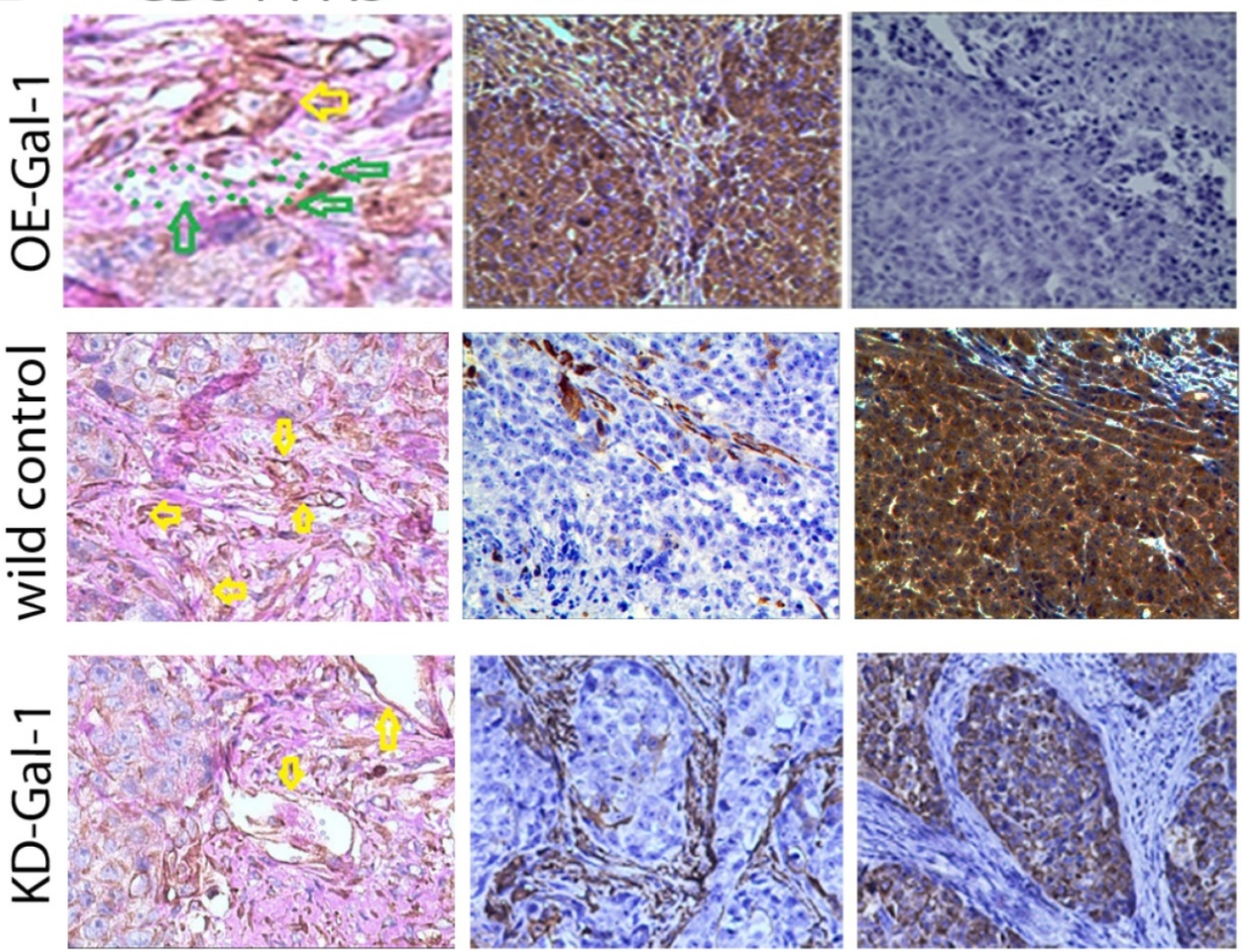

Figure 6. Manipulation of Gal-1 expression influences gastric cancer xenograft growth and VM formation (A) Gal-1 overexpression induced MGC-803 to form bigger subcutaneous xenograft Tumor weight (B) and volume (C) is expressed as the mean \pm SE. $* P<0.05, * * P<0.01, n=6$. (D) Representative Gal-1-IHC; Magnification: $\times 400$. (E) CD34-PAS staining showing endogenous cell-dependent vessels (yellow arrows) and VM (green dotted line) in OE -Gal-1, wild control and KD-Gal-1 gastric cancer xenograft, and immunostaining shown vimentin and E-cadherin in those samples. Original magnification: $\times 400$ 

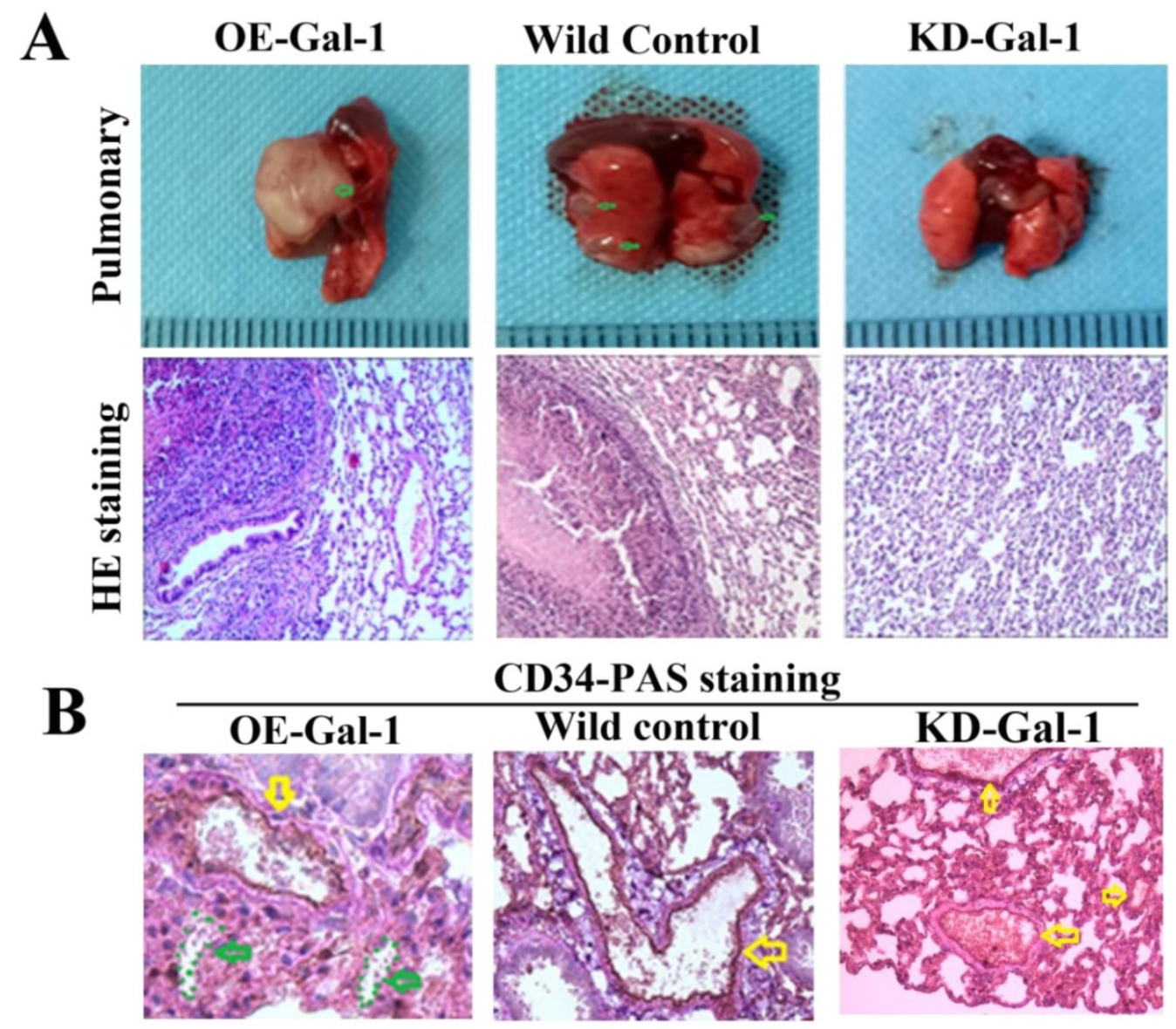

\section{CD34-PAS staining}
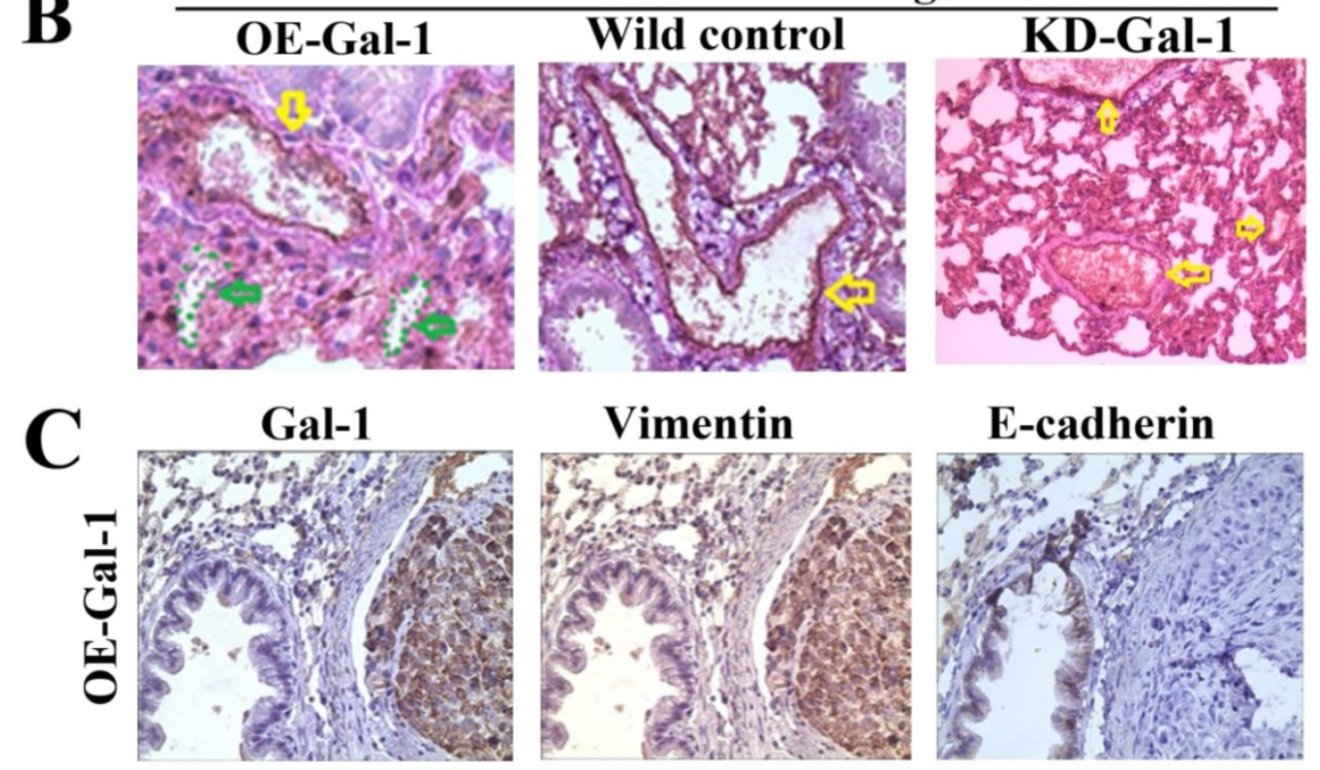

Figure 7. Manipulation of Gal-1 expression influences MGC-803 GC cells pulmonary metastases and VM formation. (A) Representative images of metastasis (green arrows) in the lungs at 50 days after inoculation, representative images of HE. Original magnification: $\times 200$. Metastases were frequent in the (B) CD34-PAS staining showing endogenous cell-dependent vessels (yellow arrows) and VM (green dotted line) in pulmonary metastases. (C) Immunostaining shown Gal-1, vimentin and E-cadherin in pulmonary metastases. Magnification: $\times 400$.

\section{Discussion}

VM formation in malignant tumor tissues tend towards tumor metastasis and have poor prognosis [29-33], which might be considered as an important target for anticancer therapy. Therefore, the study of the mechanism and the treatment of VM formation are required to improve treatments for cancer. In our previous study [24], we observed that VM formation was positively associated with the expression of Gal-1 in primary GC tissue. However, whether Gal-1 promotes VM and its mechanisms were unknown. The present study aimed to determine the role of Gal-1 in VM formation in GC.
To explore whether VM formation is associated with Gal-1 in vitro, we successfully constructed two lentiviruses for overexpression and knockdown of Gal-1, which were transduced into MGC-803 cells, separately. Overexpression of Gal-1 promoted the proliferation, invasion, migration, and VM formation of MGC-803 cells. Meanwhile knockdown Gal-1 expression in MGC-803 decreased the proliferation, invasion, migration, and VM formation of the cells. Previous studies indicated that Gal-1 promotes multiple metastatic processes via adhesion of tumor cells to the extracellular matrix, binding of cellular matrix glycoproteins, and enhancing proteolytic enzyme pathways [34, 35]. In this study, we observed 
that Gal-1 enhanced the proliferation, invasion, migration, and VM formation ability of GC cells and promote the growth and metastasis of xenograft tumors in nude mice, which agreed with previous findings.

In the in vivo experiment, we observed that high expression of Gal-1 in MGC-803 cells not only promoted tumor growth, but also promoted pulmonary metastases in nude mice. Furthermore, we found that the xenograft tumors of overexpression group showed more VM formation. Previous studies reported that there was a junction between endothelial-lined blood vessels and VM formation in tumor tissues [36, 37]. Through this junction, tumor cells lining the VM surface could directly participate in blood circulation, significantly increasing their transfer opportunities. This finding could explain the mechanism of Gal-1's promotion of metastasis in GC through the formation of VM.

Gal-1 can be detected in a variety of malignant tumors, and a high level of Gal-1 was linked to poor prognosis where Gal-1 was shown to induce EMT[16-22, 34, 35, 38]. In addition, many studies showed that EMT is an essential step in VM formation[26-28]. Therefore, we hypothesized that Gal-1 promotes the formation of VM in GC through EMT. To test this hypothesis, we established a classic EMT model for MGC-803 cells by treating them with TGF- $\beta 1$. Interestingly, we found that the expression of Gal-1 was significantly increased in the EMT model, and that Gal-1 overexpression inhibited the expression of the epithelial marker E-cadherin and promoted the expression of the mesenchymal marker vimentin in MGC-803 cells. In clinical specimens, we found that the IHC score of Gal-1 was associated with EMT-related markers in GC tissue. Furthermore, we found Gal-1 formed VM not only in GC cells when cultured in Matrigel, but also in xenograft tumors and GC specimens. The in vivo and in vitro results suggested that Gal-1 expression is related to EMT markers expression and VM formation. Taken together, our results suggest that Gal-1 may promotes EMT and forms VM in GC. Furthermore, we found that a knockdown of Gal-1 suppressed the expression of EMT markers stimulated by TGF- $\beta 1$. The results suggest that the Gal-1 / TGF- $\beta 1$ axis may be involved in Gal-1-mediated EMT in GC and Gal-1 may promote the EMT in GC by enhancing TGF- $\beta$ signaling, which is consistent with findings in a previous study[39] .

Paz A, et al.[40] found that Gal-1 promotes $\mathrm{H}-$ Ras activation intracellularly, and Ras signaling can enhance Sonic hedgehog $(\mathrm{SHH})$ expression, leading to activation of Hedgehog (Hh) signaling. Gliomaassociated oncogene -1 (Gli-1) is activated by $\mathrm{Hh}$ signaling to induce and promote EMT of GC [41].
Therefore, -Gal-1 may alsopromotes EMT in GC via the Ras/Hh/Gli-1 signaling pathway.So the specific molecular mechanisms of Gal-1's promotion VM through EMT are needed further study.

Previous studies showed that Gal-1 and VM were identified in multivalent malignant tumors [7-13, 16-19]. Gal-1 was also shown to induce EMT [16-22, 34, 35, 38], while EMT is an essential step in VM formation [26-28]. However, whether Gal-1 also promotes the VM formation in other tumors remains to be investigated. Further experiment is planned to explore the hypothesis that Gal-1 promote EMT and VM in GC or other tumors.

\section{Conclusions}

In conclusion, we implemented substantial experiments to explore the mechanism of Gal-1 induced VM in GC. We confirmed that Gal-1 promotes GC progression through VM formation by activating the EMT pathway. Our results will provide therapeutic targets for the development of new drugs to treat GC.

\section{Abbreviations}

EMT: epithelial-to-mesenchymal transition; FBS: fetal bovine serum; Gal-1: galectin-1; GC: gastric cancer; GFP: green fluorescent protein; Gli-1: Glioma-associated oncogene-1; H\&E: hematoxylineosin; Hh: Hedgehog; HRP: Horseradish peroxidase; IHC: immunohistochemistry; KD-Gal-1: Gal-1 knock-down; MOI: multiplicity of infection; OE-Gal-1: overexpressing Gal-1; PBS: phosphate-buffered saline; qRT-PCR: quantitative real-time PCR; PAS: Periodic acid-Schiff; RPMI: Roswell Park Memorial Institute medium; SDS-PAGE: sodium dodecyl sulfatepolyacrylamide gel electrophoresis; $\mathrm{SHH}$ : Sonic hedgehog; VIM: Vimentin; VM: Vasculogenic mimicry; WC: wild-type control.

\section{Acknowledgments}

This work was supported in part by China Postdoctoral Science Foundation (grant number 2018M632400), Science and Technology Support Program (Social Development) Project of Taizhou City: TS201824.

\section{Competing Interests}

The authors have declared that no competing interest exists.

\section{References}

1. Zhu QH, Wang YY, Chen YF, et al. Effect of interleukin-17A and interleukin-17F gene polymorphisms on the risk of gastric cancer in a Chinese population. Gene. 2014; 537(2):328-332.

2. Long ZW, Yu HM, Wang YN, et al. Association of IL-17 polymorphisms with gastric cancer risk in Asian populations. World J Gastroenterol. 2015; 21(18):5707-5718. 
3. Mawalla B, Yuan X, Luo X, Chalya PL. Treatment outcome of anti-angiogenesis through VEGF-pathway in the management of gastric cancer: a systematic review of phase II and III clinical trials. BMC Res Notes. 2018; 11(1):21.

4. Yu J, Zhang Y, Leung LH,et al. Efficacy and safety of angiogenesis inhibitors in advanced gastric cancer: a systematic review and meta-analysis. J Hematol Oncol. 2016; 9(1):111

5. Allegra CJ, Yothers G, O'Connell MJ, et al. Phase III trial assessing bevacizumab in stages II and III carcinoma of the colon: results of NSABP protocol C-08. J Clin Oncol. 2010; 29(1):11-16.

6. Maniotis AJ, Folberg R, Hess A, et al. Vascular channel formation by human melanoma cells in vivo and in vitro: vasculogenic mimicry. Am J Pathol. 1999; 155(3):739-752.

7. Sun W, Fan YZ, Zhang WZ, Ge CY. A pilot histomorphology and hemodynamic of vasculogenic mimicry in gallbladder carcinomas in vivo and in vitro. J Exp Clin Cancer Res. 2011; 30(1):46.

8. Sun D, Sun B, Liu T, et al. Slug promoted vasculogenic mimicry in hepatocellular carcinoma. J Cell Mol Med. 2013; 17(8):1038-1047.

9. Gao S, Fan C, Huang H, et al. Effects of HCG on human epithelial ovarian cancer vasculogenic mimicry formation in vivo. Oncol Lett. 2016; 12(1):459-466

10. Williamson SC, Metcalf RL, Trapani F, et al. Vasculogenic mimicry in small cell lung cancer. Nat Commun. 2016; 7:13322.

11. Angara K, Borin TF, Arbab AS. Vascular Mimicry: A Novel Neovascularization Mechanism Driving Anti-Angiogenic Therapy (AAT) Resistance in Glioblastoma. Transl Oncol. 2017; 10(4):650-660.

12. Wei HL, Wang FH, Wang Y, et al. Verteporfin suppresses cell survival, angiogenesis and vasculogenic mimicry of pancreatic ductal adenocarcinoma via disrupting the YAP-TEAD complex. Cancer Sci. 2017; 108(3):478-487.

13. Song YY, Sun LD, Liu ML, et al. STAT3, p-STAT3 and HIF-1a are associated with vasculogenic mimicry and impact on survival in gastric adenocarcinoma. Oncol Lett. 2014; 8(1):431-437.

14. Racordon D, Valdivia A, Mingo G, et al. Structural and functional identification of vasculogenic mimicry in vitro. Sci Rep. 2017; 7(1):6985.

15. Qiao Ll, Liang N, Zhang JD, et al. Advanced research on vasculogenic mimicry in cancer. J Cell Mol Med. 2015; 19(2):315-326.

16. Cousin JM, Cloninger MJ. The Role of Galectin-1 in Cancer Progression, and Synthetic Multivalent Systems for the Study of Galectin-1. Int J Mol Sci. 2016; 17(9):1566.

17. Carlsson MC, Cederfur C, Schaar V, et al. Galectin-1-binding glycoforms of haptoglobin with altered intracellular trafficking, and increase in metastatic breast cancer patients. PLoS One. 2011; 6(10):e26560.

18. Chen R, Pan S, Ottenhof NA, et al. Stromal galectin-1 expression is associated with long-term survival in resectable pancreatic ductal adenocarcinoma. Cancer Biol Ther. 2012; 13(10):899-907.

19. Martínez-Bosch N, Fernández-Barrena MG, Moreno M, et al. Galectin-1 drives pancreatic carcinogenesis through stroma remodeling and Hedgehog signaling activation. Cancer Res. 2014; 74(13):3512-3524.

20. Griffioen AW, Thijssen VL. Galectins in tumor angiogenesis. Ann Transl Med. 2014; 2(9):90.

21. Thijssen VL, Griffioen AW. Galectin-1 and -9 in angiogenesis: a sweet couple. Glycobiology. 2014; 24(10):915-920.

22. Noda Y, Kishino M, Sato S, et al. Galectin-1 expression is associated with tumour immunity and prognosis in gingival squamous cell carcinoma. J Clin Pathol. 2017; 70(2):126-133.

23. Méndez-Huergo SP, Blidner AG, Rabinovich GA. Galectins: emerging regulatory checkpoints linking tumor immunity and angiogenesis. Curr Opin Immunol. 2017; 45(4):8-15.

24. You $\mathrm{XL}$, Wang YJ, Wu J , et al. Prognostic significance of galectin-1 and vasculogenic mimicry in patients with gastric cancer. Onco Targets Ther. 2018; 11:3237-3244.

25. Chen DH, Zhou HH, Liu GY, et al. SPOCK1 promotes the invasion and metastasis of gastric cancer through Slug-induced epithelial-mesenchymal transition. J Cell Mol Med. 2018; 22(2):797-807.

26. Liu QQ, Qiao LL, Liang $\mathrm{N}$, et al. The relationship between vasculogenic mimicry and epithelial-mesenchymal transitions. J Cell Mol Med 2016; 20(9):1761-1769

27. Wang $W$, Lin $P$, Sun $B C$, et al. Epithelial-mesenchymal transition regulated by EphA2 contributes to vasculogenic mimicry formation of head and neck squamous cell carcinoma. Biomed Res Int. 2014; 2014:803914.

28. Han C, Sun BC, Zhao XL, et al. Phosphorylation of STAT3 Promotes Vasculogenic Mimicry by Inducing Epithelial-to-Mesenchymal Transition in Colorectal Cancer. Technol Cancer Res Treat. 2017; 16(6):1209-1219.

29. Bissanum R, Lirdprapamongkol K, Svasti J,et al. The role of WT1 isoforms in vasculogenic mimicry and metastatic potential of human triple negative breast cancer cells. Biochem Biophys Res Commun. 2017; 494(1-2):256-262.

30. Han GS, Li YN, Cao YQ, et al. Overexpression of leptin receptor in human glioblastoma: Correlation with vasculogenic mimicry and poor prognosis. Oncotarget. 2017; 8(35):58163-58171.

31. Chen LX, He YJ, Sun SZ, et al. Vasculogenic mimicry is a major feature and novel predictor of poor prognosis in patients with orbital rhabdomyosarcoma. Oncol Lett. 2015; 10(3):1635-1641.

32. Zhu B, Zhou L, Yu L, et al. Evaluation of the correlation of vasculogenic mimicry, ALDH1, KAI1 and microvessel density in the prediction of metastasis and prognosis in colorectal carcinoma. BMC Surg. 2017;17(1):47.
33. Li M, Gu YJ, Zhang ZG, et al. Vasculogenic mimicry: a new prognostic sign of gastric adenocarcinoma. Pathol Oncol Res. 2010; 16(2):259-266.

34. Roda O, Ortiz-Zapater E, Martinez-Bosch N, et al. Galectin-1 is a novel functional receptor for tissue plasminogen activator in pancreatic cancer. Gastroenterology. 2009; 136(4):1379-1390.

35. Wu MH, Hong TM, Cheng HW, et al. Galectin-1-mediated tumor invasion and metastasis, up-regulated matrix metalloproteinase expression, and reorganized actin cytoskeletons. Mol Cancer Res. 2009; 7(3):311-318.

36. Kobayashi H, Shirakawa K, Kawamoto S, et al. Rapid accumulation and internalization of radiolabeled herceptin in an inflammatory breast cancer xenograft with vasculogenic mimicry predicted by the contrast-enhanced dynamic MRI with the macromolecular contrast agent G6-(1B4M-Gd)(256). Cancer Res. 2002; 62(3):860-866.

37. Clarijs R, Otte-Höller I, Ruiter DI, de Waal RM. Presence of a fluid-conducting meshwork in xenografted cutaneous and primary human uveal melanoma. Invest Ophthalmol Vis Sci.. 2002; 43(4):912-918.

38. Zhang PF, Li KS, Shen $\mathrm{YH}$, et al. Galectin-1 induces hepatocellular carcinoma EMT and sorafenib resistance by activating FAK/PI3K/AKT signaling. Cell Death Dis. 2016; 7:e2201

39. Zheng LY, Xu C, Guan ZH, et al. Galectin-1 mediates TGF-beta-induced transformation from normal fibroblasts into carcinoma-associated fibroblasts and promotes tumor progression in gastric cancer. Am J Transl Res. 2016; 8(4):1641-1658

40. Paz A, Haklai R, Elad-Sfadia G, et al. Galectin-1 binds oncogenic H-Ras to mediate Ras membrane anchorage and cell transformation. Oncogene. 2001; 20(51):7486-7493

41. Chong Y, Tang D, Gao J, et al. Galectin-1 induces invasion and the epithelial-mesenchymal transition in human gastric cancer cells via non-canonical activation of the hedgehog signaling pathway. Oncotarget. 2016; 7(50):83611-83626. 